\title{
Transatlantica
}

Revue d'études américaines. American Studies Journal

\section{Hawthorne et ses métalepses}

\section{Guillaume Tanguy}

\section{(2) OpenEdition}

Journals

Édition électronique

URL : http://journals.openedition.org/transatlantica/1601

DOI : 10.4000/transatlantica.1601

ISSN : 1765-2766

Éditeur

AFEA

\section{Référence électronique}

Guillaume Tanguy, «Hawthorne et ses métalepses», Transatlantica [En ligne], 1 | 2007, mis en ligne le 03 août 2007, consulté le 29 avril 2021. URL : http://journals.openedition.org/transatlantica/1601 ; DOI : https://doi.org/10.4000/transatlantica.1601

Ce document a été généré automatiquement le 29 avril 2021.

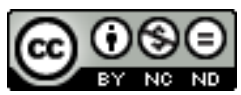

Transatlantica - Revue d'études américaines est mis à disposition selon les termes de la licence Creative Commons Attribution - Pas d'Utilisation Commerciale - Pas de Modification 4.0 International. 


\title{
Hawthorne et ses métalepses
}

\author{
Guillaume Tanguy
}

«[... the portrait, frame and all, tumbled
suddenly from its position,
and lay face downward on the floor. "
Nathaniel Hawthorne, The House of the Seven

Gables)

«La visibilité est un piège. »

(Michel Foucault, Surveiller et punir)

« [Le] contact même imaginaire immunise contre la

mort [...]. »

(Julien Gracq, La littérature à l'estomac)

1 À la lecture de la critique consacrée à Nathaniel Hawthorne, le lecteur ne manque pas d'être frappé par un certain nombre d'idées récurrentes. L' « interstice», le «territoire du presque », l'« analogie » entre l'activité du narrateur et celle de ses personnages, ou encore la volonté de "franchir les murailles », de passer " d'un régime à l'autre » sont autant de marques du texte hawthornien. La modernité de l'œuvre est inséparable de sa "porosité", qui semble annoncer le régime d'instabilité actuel ${ }^{1}$. Une remarque de Jorge Luis Borges permet de synthétiser ces diverses analyses :

Hawthorne était homme de perpétuelle et curieuse imagination, mais réfractaire, disons-le ainsi, à la pensée. [I]l pensait par images, par intuitions, comme pensent habituellement les femmes, non selon un mécanisme dialectique. [Le thème de ses contes] est la coïncidence ou la confusion du plan esthétique et du plan commun, de la réalité et de l'art. [...] De tels jeux, de telles confluences momentanées du monde imaginaire et du monde réel - sont, ou nous semblent, modernes. [...] Ces contacts de l'imaginaire et du réel plaisaient à Hawthorne, ce sont reflets et duplications de l'art. (Borges 83-86)

2 Borges détecte chez Hawthorne une prédilection marquée pour une figure qu'il affectionnait lui-même particulièrement: la métalepse ${ }^{2}$. Le propos de cet article est, dans la lignée de ces intuitions, de montrer que la spécificité du fait poétique hawthornien réside précisément dans son caractère métaleptique. Notre analyse 
examinera plusieurs textes avant de se concentrer sur The Scarlet Letter, où le traitement de cette figure est le plus achevé.

3 Commençons par rappeler la définition de la figure. On appelle métalepse toute transgression délibérée d'un seuil narratif, tout franchissement de la "frontière mouvante mais sacrée entre deux mondes: celui où l'on raconte, celui que l'on raconte » (Figures III 245), soit que le personnage « sorte » du cadre (Genette), soit qu'il le «brise » (Malina). «Genette defines metalepsis as 'any intrusion by the extradiegetic narrator or narratee into the diegetic universe (or by diegetic characters into a metadiegetic [...] universe, etc.), or the inverse » (Malina 3). Ou encore : « un narrateur extradiégétique entre soudainement en relation directe avec l'un des personnages diégétiques" (Cohn). S'il est facile de décrire le phénomène, son interprétation, en revanche, suscite des divergences. Quel effet cette " effraction de la clôture textuelle » (Wagner 247) produit-elle? Si Malina rejoint Genette sur la définition du trope, sa lecture de l'impact sur le lecteur semble plus restrictive. La métalepse, selon elle, est une figure subversive, liée à la modernité et à la postmodernité (elle l'étudie chez Beckett, Christine Brooke-Rose et Angela Carter). Le texte (post)moderne l'emploie pour subvertir le concept de réel, déconstruire la hiérarchie des systèmes de savoir, et favoriser le brouillage entre réel et fiction ${ }^{3}$. Malina y voit une intrusion qui menace le lecteur, un phénomène agressif fait de "cadrages, décadrages et recadrages violents » :

[The] term 'intrusion' haunts my reading of the dynamic. For regardless of the fluidity in which a given metaleptic move cloaks itself, it necessarily violates the structure of the narrative and disrupts the reading process that relies on that structure for its constituting of meaning. The pairing of metalepsis with the physical violation staged in many of Angela Carter's novels, for example, foregrounds the violence implicit in such intrusions. (Malina 4)

4 La métalepse choque le lecteur parce qu'elle franchit une barrière ontologique, donc hiérarchique. Elle s'inscrirait dans une "vaste entreprise de contestation métatextuelle» (Wagner 241). Genette, lui, propose une interprétation apparemment plus large de la figure, comme le rappelle Frank Wagner: "Genette précise que le trouble généré par les métalepses 'ne peut relever que de l'humour [...] ou du fantastique [...], ou de quelque mixte des deux [...], à moins qu'il ne fonctionne comme une figure de l'imagination créatrice'» (Wagner 249). Néanmoins, Genette insiste, lui aussi, sur l'inquiétude, ou du moins le trouble inhérent à la métalepse :

D'où l'inquiétude si justement désignée par Borges : 'De telles inversions suggèrent que si les personnages d'une fiction peuvent être lecteurs ou spectateurs, nous, leurs lecteurs ou spectateurs, pouvons être des personnages fictifs.' Le plus troublant dans la métalepse est bien dans cette hypothèse inacceptable et insistante, que l'extradiégétique est peut-être toujours déjà diégétique, et que le narrateur et ses narrataires, c'est-à-dire vous et moi, appartenons peut-être encore à quelque récit. (Figures III 245)

5 Cet article tentera de montrer que la métalepse peut se révéler ambivalente selon qu'elle constitue une intrusion indiscrète, ou qu'elle suscite au contraire une relation identificatoire, voire amoureuse - cette seconde valeur étant probablement la plus spécifiquement hawthornienne. Par conséquent, on insistera moins sur le caractère « inacceptable » ou « inquiétant » de la figure que sur sa dimension troublante, au sens où elle produit chez le lecteur un émoi, et favorise les perceptions troubles, compromettant délibérément la bonne visibilité. Le cas de The Scarlet Letter montrera enfin que l'écriture métaleptique a également des implications politiques, et exprime la résistance à une certaine conception de l'autorité. La réflexion de Michel Foucault sur 
la prison et la discipline permettra de dégager, par contraste, la signification de l'esthétique hawthornienne. L'art du trouble que celle-ci met en place a pour visée la subversion des mécanismes disciplinaires et des carcans de toutes sortes. Loin de surveiller et de punir, elle se veut libératrice et salvatrice.

Métalepse et cadre

6 La notion de cadre, envisagée tout d'abord sur le plan biographique, permettra d'engager l'analyse. Nathaniel Hawthorne avait beau affectionner les «structures emboîtées » et les «organismes gigognes" (Normand 17), il cherchait surtout à échapper aux systèmes contraignants quels qu'ils soient. La première prison fut la chambre, lieu de l'immobilité forcée. A cause d'une blessure au pied survenue en 1813, le jeune Nathaniel cessa de se rendre à l'école pendant deux ans et quatre mois. Son existence fut circonscrite aux murs de sa chambre :

Les limites de l'univers de Nathaniel vont être, pendant des mois, celles de la maison Manning. [...] La plupart du temps, c'est l'allongement. Par la fenêtre, des toits, dont celui de la maison natale, en forme de croupe. Le petit invalide rêve peut-être encore de l'immense grenier où il jouait à s'inventer des peurs, des fantômes. [Il se sent] isolé, frustré de contacts avec l'extérieur [...]. (Normand 25-26)

Dès qu'il retrouva sa mobilité, l'enfant chercha à assouvir sa soif d'errance. Le séjour dans la propriété familiale de Raymond lui permit de sillonner pendant neuf mois la campagne du Maine autour du lac Sebago. Une autre période de claustration, autoinfligée celle-là, intervint pendant les « douze années solitaires " qu'il passa à lire et à écrire dans sa chambre de la maison familiale à Salem. Claustration nécessaire pour conquérir la gloire, mais claustration néanmoins abhorrée : «In this dismal chamber FAME was won ", écrit-il le 31 août 1836.

8 Étudiant, Hawthorne n'affectionnait pas davantage la discipline de la vie universitaire (il évitait les exercices de déclamation imposés par le Président de Bowdoin College); écrivain, il avait une aversion pour les cadres théoriques: "Sophia [Hawthorne] claimed that her husband was 'without theories of any kind'» (Millington 11). Aussi resta-t-il en marge du courant optimiste qui devait inspirer le Transcendentalisme, et ironisa-t-il sur les intellectuels de son temps, notamment Emerson et Fuller. Dans « The Old Manse » (1846), il présente la spéculation intellectuelle comme une prison : «Grayheaded theorists [...] had finally imprisoned them in an iron frame-work [...]» (Tales and Sketches 1146).

9 Ces rappels biographiques aident à cerner le tempérament de l'écrivain, mais puisque la métalepse est un phénomène narratif, il faut maintenant se tourner vers les textes. Pour décrire le fonctionnement de la figure, on peut commencer par une remarque négative : la métalepse n'est pas un simple geste rhétorique, et Hawthorne n'est pas Balzac. Genette fait la remarque suivante :

Lorsque Balzac [...] écrit 'Pendant que le vénérable ecclésiastique monte les rampes d'Angoulême, il n'est pas inutile d'expliquer, etc.', chacun traduit que le romanciernarrateur suspend simplement le récit pour donner à son lecteur quelques explications utiles à la compréhension de son intrigue. [C'est une] fiction figurale [qui] associe simplement le lecteur [...] à l'acte de narration. (Métalepse 22-23)

10 De même, si l'on se reporte à Uncle Tom's Cabin, roman presque contemporain de The Scarlet Letter, on constate que la métalepse n'est qu'un geste discursif (on songe par exemple au chapitre IV, où la narratrice "laisse" la tante Chloé vaquer à ses occupations pendant qu'elle achève de décrire la case) ${ }^{4}$. En somme, chez Harriet 
Beecher Stowe comme chez Balzac, la métalepse n'a qu'une fonction de "régie " (Wagner 238). Au contraire, la plupart des textes que l'on va examiner confèrent à la métalepse une véritable fonction esthétique.

Amorces métaleptiques

11 Avant d'envisager The Scarlet Letter, il paraît judicieux de procéder à un rapide balayage de l'œuvre de Hawthorne: on trouve ainsi, dans une première catégorie de textes (antérieurs et postérieurs au roman de 1850), des amorces métaleptiques qui instaurent un échange narratif, même si leur fonction demeure marginale. Catégorie à lui seul, The Blithedale Romance explore en revanche les dangers inhérents à l'impulsion métaleptique. C'est seulement après avoir mis en évidence ces valeurs opposées de la figure qu'on sera en mesure d'apprécier la complexité de The Scarlet Letter.

Le nombre de textes concernés par la métalepse est significatif, et leur lecture successive fait apparaître une gradation esthétique. On trouve d'abord des figures qui, comme chez Balzac et Stowe, n'ont qu'une fonction de régie. Dans "Mrs. Hutchinson » (1830), par exemple, le narrateur feint d'entrer dans la pièce où se déroule l'action, afin de décrire les personnages : «We also will go in, edging through the thronged doorway to an apartment which occupies the whole breadth of the house. [...] Let us look round upon the hearers " (Tales and Sketches 20). Dans "The May-Pole of Merry Mount " (1836), le narrateur regrette de s'être fourvoyé dans une digression historique, et constate à regret que la nuit tombe sur son récit : « Alas! we have delayed too long and must darken our tale too suddenly! » (Tales and Sketches 366). Les personnages semblent pâtir de sa lenteur. La situation s'inverse dans The House of the Seven Gables (1851), où le narrateur, par pudeur métaleptique, doit attendre que Hepzibah ait terminé sa toilette avant de commencer à la décrire :

Far from us be the indecorum of assisting, even in imagination, at a maiden lady's toilet! Our story must therefore await Miss Hepzibah at the threshold of her chamber [...]. The maiden lady's devotions are concluded. Will she now issue forth over the threshold of our story? [...] Now, she is almost ready. Let us pardon her one other pause [...]. (The House of the Seven Gables 30-31)

Mais, dans ces deux derniers cas, la métalepse acquiert déjà une valeur esthétique, car elle participe de la tonalité facétieuse du récit. Plus loin, au chapitre XII, le narrateur imagine son lecteur en train d'épier les personnages, Phoebe et Holgrave, à travers les fentes de la clôture : "But, had you peeped at them through the chinks of the gardenfence, the young man's earnestness might have led you to suppose that he was making love to the young girl!» (182) Un autre exemple intervient au chapitre VII, où le portrait du colonel Pyncheon semble sortir de son cadre : « [Clifford's eyes happened to rest] on the face of the old Puritan, who, out of his dingy frame and lustreless canvass, was looking down on the scene like a ghost, and a most ill-tempered and ungenial one (The House of the Seven Gables 110). Ici, pour reprendre les mots de Genette, «Le tableau s'anime. [...] Le personnage sort du cadre, s'émancipe de son médium mimétique, et passe de l'univers représenté à l'univers où figurait jusqu'alors sa représentation " (81-89). Dans ces deux exemples, on voit pointer une autre caractéristique : le caractère intrusif, voir agressif de la figure. C'est d'ailleurs pour cette raison que le narrateur ne permet pas à l'ancêtre malveillant de sortir véritablement du cadre. La figure prend une tout autre valeur dans «Wakefield» (1835), où le conteur fait mine de suivre le personnage éponyme : «We must hurry after him [...]. Let us follow close at his heels, therefore, until, after several superfluous turns and doublings, we find him comfortably established by the fireside of a small apartment, previously bespoken » (Tales and 
Sketches 292). Ou encore: "We will not follow our friend across the threshold [...]» (298). Si le procédé rappelle celui de "Mrs. Hutchinson", il s'est indéniablement enrichi : en faisant mine de prendre part à l'action, le conteur cherche à entrer en contact avec le personnage. La distance qu'il cherche à abolir entre lui et Wakefield est narrative, mais aussi affective. Un phénomène semblable survient dans la Préface de Mosses from an old Manse, où Hawthorne présente au lecteur, son «invité » («my guest »), le vieux presbytère :

Has the reader gone wandering, hand in hand with me, through the inner passages of my being, and have we groped together into all its chambers, and examined their treasures or their rubbish? Not so. We have been standing on the green sward, but just within the cavern's mouth, where the common sunshine is free to penetrate [...]. So far as I am a man of really individual attributes, I veil my face. (Tales and Sketches 1147)

14 À quel moment la communication métaleptique devient-elle inquisition ? Le but du passage est moins de répondre à cette question que de mettre en évidence les deux faces opposées de la figure - la communion et l'intrusion - et de proposer un gardefou, le voile hawthornien. La relation qui se noue ne doit pas dé-voiler l'intimité de l'énonciateur autobiographique de façon indiscrète, mais s'épanouir à la faveur d'une transparence obscure.

Le revers de la Romance. Coverdale ou la métalepse cryptée

Ce pacte de non-agression est remis en cause par The Blithedale Romance, où s'actualise la menace pointée dans "The Old Manse». Le premier signe est probablement l'ambiguïté inquiétante du statut narratif du protagoniste. Robert $\mathrm{S}$. Levine note à ce propos : "As presented in the novel, Coverdale is simultaneously an insider and an outsider, a fully energetic participant and a more ironic retrospective narrator [...] » (Millington 213). Au début du récit, Coverdale participe activement à l'entreprise communautariste. Il y a donc d'un côté le Miles-actant et de l'autre le Miles-narrateur rétrospectif - configuration qui, en soi, n'est pas proprement métaleptique. Mais, peu à peu, le jeune célibataire se désengage de la communauté, puis se réfugie dans son ermitage, d'où il voit tout sans être vu (situation panoptique par excellence). Au chapitre XVI, il quitte la colonie, renouant avec son existence citadine. Dès lors, il n'est plus participant dans le récit qu'il décrit, mais spectateur, et même auteur: en décrivant les événements, il compose une œuvre d'art. Il est d'ailleurs significatif que, depuis sa chambre d'hôtel où il épie Zenobia, Westervelt et Priscilla, il décrit ce qu'il voit comme une pièce de théâtre : la fenêtre, les rideaux qui l'entourent et l'éclairage aveuglant constituent à la fois un cadrage visuel et une mise en scène. Cette transformation en écrivain n'échappe pas à Zenobia ${ }^{5}$. La nature indiscrète du regard de Coverdale montre que celui-ci cherche à lever le voile hawthornien-à devenir inquisiteur - même si cette tentative est contrariée par le geste de la jeune femme : «[She let] down a white linen curtain between the festoons of the damask one. It fell like the drop-curtain of a theatre, in the interval between the acts » (The Complete novels 533). Fuyant l'intrusion métaleptique, Zenobia abrite son Moi intime derrière le rideau.

16 Un autre franchissement du seuil narratif a lieu vers la fin du récit. Mais cette pulsion métaleptique est soigneusement dissimulée par le narrateur, Coverdale le bien nommé, ce qui fait la spécificité du roman. Pour détecter la métalepse, le lecteur doit commencer par relever deux métaphores proleptiques: depuis sa chambre d'hôtel, Coverdale décrit la vie autour de lui comme un cours d'eau où il répugne à plonger, préférant rester sur la berge. Puis il évoque le roman qu'il lit, et assimile l'intrigue 
ennuyeuse à un ruisseau léthargique ${ }^{6}$. Ces images aident le lecteur à mieux appréhender le chapitre XXVII, moment culminant où l'on apprend la nouvelle de la mort de Zenobia, et où Coverdale, Hollingsworth et Silas Foster partent à la recherche de la jeune femme. Avant de retrouver le corps, ils aperçoivent une de ses chaussures. Silas Foster la sort de la boue, et Coverdale s'en saisit, puis la rince; il gardera toujours ce soulier avec lui : "I dabbled it in the water, to rinse off the mud, and have kept it ever since » (576). Le soulier passe de la diégèse au récit ${ }^{7}$, sortant ainsi de son cadre. Mais c'est surtout le statut du jeune homme qui importe ici: on se souvient que Coverdale a été comparé plus tôt à un auteur, et qu'il comparait lui-même la fiction à un cours d'eau. Or il se retrouve maintenant sur un petit ruisseau, ce qui suggère qu'il a franchi un seuil narratif. De plus, son comportement diffère de celui de ses acolytes - signe qu'il ne se situe pas sur le même niveau narratif: Hollingsworth et Foster sont actifs, alors qu'il se montre très passif. Eux tiennent des outils pour repêcher le corps, lui tient la barre qui dirige la barque. Eux s'activent vigoureusement pour sortir le corps de l'eau (" laid hold of the body », " grappled with it » [577]), lui contemple la scène ( I I steered towards the bank, gazing all the while at Zenobia » [577]), et n'a aucun contact tactile avec le corps. Enfin, on note la remarque suivante : "'Well, Miles Coverdale,' said Foster, 'you are the helmsman. How do you mean to manage this business? » (576, nos italiques). Cette interrogation peut se lire au deuxième degré : les protagonistes demandent au narrateur comment il compte résoudre la situation et les aider à retrouver le corps. Contrairement au début du récit, où Coverdale était à la fois personnage-actant et narrateur rétrospectif, il n'est ici plus guère que narrateur - mais un narrateur qui, fasciné par son propre récit, désire franchir le seuil de la représentation et s'immiscer dans la diégèse. Dans la mesure où, ici, le narrateur mène la barque au propre et au figuré, on peut interpréter l'épisode comme une entrevue entre le romancier et ses personnages.

Il apparaît d'ores et déjà que la métalepse n'est pas un phénomène marginal, mais une idiosyncrasie hawthornienne. Hormis les cas où elle a une fonction de régie, elle fait partie intégrante de l'esthétique romanesque. Tous les exemples recensés plus haut ont pour but de mettre en contact la diégèse avec l'instance narrative. Ce contact est la plupart du temps bienveillant, à l'exception notoire de The Blithedale Romance. Dans ce roman, on décèle une froideur marquée entre Coverdale et Foster ${ }^{8}$. Surtout, c'est la fascination de Coverdale pour Zenobia qui inquiète: sa curiosité est proche du voyeurisme, et se redouble d'une absence de sympathie humaine ${ }^{9}$. Au chapitre XXIV, ses anciens camarades surprennent le jeune homme en train d'épier leur mascarade, tel un intrus irrévérencieux (“'Some profane intruder!' " [563]). Coverdale, lorsqu'il cherche à pénétrer dans l'intimité de ceux qui l'entourent, est parfois bien proche d'Ethan Brand, incarnation du " Péché Impardonnable ». Dépourvu de sympathie, son regard apparait comme une forme de compensation immorale. C'est sans doute d'ailleurs la raison pour laquelle, dans le roman de 1852, la métalepse est cryptée par le statut narratif complexe de Coverdale: le narrateur-personnage est en fait un narrateur qui s'immisce métaleptiquement, un sinistre passe-muraille (l'adjectif "border-straddling " [Malina 12] serait pertinent ici). Cette hypothèse va dans le sens de l'argumentation de Malina sur la violence latente du trope, pouvant mener, selon elle, jusqu'au sado-masochisme, au viol, à la torture, bref à la subjugation sous toutes ses formes [Malina 11-12]). Coverdale fait mine de se joindre à la communauté pour mieux l'épier à couvert. La liberté sexuelle qui règne à Blithedale le fascine, et lui permet de donner cours à ses pulsions secrètes (son attirance pour Zenobia et Priscilla, de même 
que sa fascination homoérotique pour Hollingsworth [Millington 215]), mais sans passer à l'acte. De « The Old Manse » à The Blithedale Romance, l'invité inoffensif se mue en sinistre prédateur. Dès lors, intrusion métaleptique rime avec profanation.

The Scarlet Letter : la concaténation empathique

La spécificité de The Scarlet Letter tient tout d'abord à sa position chronologique. Que l'on voie dans ce roman le chef d'œuvre de Hawthorne, comme le font de nombreux critiques, ou que l'on se range à l'avis de Borges, pour qui le génie de l'auteur se prête davantage à la forme courte (Borges, 412), il faut rappeler la position privilégiée du roman de 1850, qui se situe après les textes courts (publiés avant 1850) et au début de la phase romanesque (1850-1860). Dans The Scarlet Letter, la tendance métaleptique caractéristique des textes courts arrive à maturité : la forme romanesque lui donne davantage d'ampleur et accroît sa signification esthétique. En outre, la figure y est plus signifiante que dans les romans ultérieurs : dans The House of the Seven Gables, elle a essentiellement une fonction de régie; dans The Blithedale Romance, son caractère insidieux la rend inquiétante et, surtout, ne permet pas à l'amour de Coverdale pour Priscilla de s'épanouir. Envisagé à l'aune de la métalepse, le roman de 1850 apparaît sans conteste comme le grand texte hawthornien.

Les différences avec The Blithedale Romance et avec The House of the Seven Gables ne doivent pas faire oublier un point commun : à deux reprises, la métalepse constitue une intrusion. Lorsque, au chapitre III, Wilson interpelle Hester Prynne, le narrateur compare le prédicateur sévère à un portrait dans un frontispice. Wilson semble vouloir sortir de son cadre afin de se mêler de questions passionnelles qui, selon le narrateur, ne le regardent en rien. Il s'agit d'une infraction, au sens légal du terme, comme le souligne le terme « right» :

[Wilson] looked like the darkly engraved portraits which we see prefixed to old volumes of sermons; and had no more right than one of those portraits would have, to step forth, as he now did, and meddle with a question of human guilt, passion, and anguish. (The Scarlet Letter 48)

À nouveau, le portrait tente de sortir du cadre, et veut s'immiscer de façon indiscrète. Le même schéma se produit lors de la description de la demeure de Bellingham et des portraits qui s'y trouvent. Ces portraits semblent regarder les vivants et sortir du cadre : «[...] as if they were the ghosts, or rather the pictures, of departed worthies, and were gazing with harsh and intolerant criticism at the pursuits and enjoyments of living men " (71). Une autre forme d'intrusion a lieu lorsque Chillingworth, médecin attitré de Dimmesdale, devient subrepticement le bourreau qui le met au supplice : « He became, thenceforth, not a spectator only, but a chief actor, in the poor minister's interior world» (93). À la fois observateur du drame intérieur du jeune homme et protagoniste au sein de ce drame, il franchit la « frontière sacrée » (Genette) entre deux mondes, ce qui constitue, pour reprendre l'expression de Dimmesdale lui-même, une violation: "He has violated, in cold blood, the sanctity of a human heart» (126). Cela étant, la portée métaleptique de ces deux exemples demeure limitée. Dans le second cas, Chillingworth ne change pas de statut narratif au sein du récit (contrairement à Miles Coverdale) : qu'il soit médecin ou bourreau, il demeure personnage. Dans le premier cas, la métalepse est une image (le portrait semble sortir du cadre) et, de surcroît, le narrateur la condamne comme immorale, refusant ainsi de la prendre à son compte. 
21 Les autres métalepses du roman, au nombre de trois, sont d'une autre nature. Elles cessent d'avoir une valeur négative, et interviennent toutes à des moments-clés : la première a lieu dans «The Custom-House ", juste après la découverte de la lettre dans le grenier de la Douane. Le narrateur de «The Custom-House » constate alors que son statut d'Inspecteur paralyse ses facultés d'écrivain. Cette inactivité artistique attire sur lui les foudres de ses personnages :

My imagination was a tarnished mirror. It would not reflect, or only with miserable dimness, the figures with which I did my best to people it. The characters of the narrative would not be warmed and rendered malleable by any heat that I could kindle at my intellectual forge. They would take neither the glow of passion nor the tenderness of sentiment, but retained all the rigidity of dead corpses, and stared me in the face with a fixed and ghastly grin of contemptuous defiance. "What have you to do with us?" that expression seemed to say. "The little power you might have once possessed over the tribe of unrealities is gone! You have bartered it for a pittance of the public gold. Go, then, and earn your wages!" In short, the almost torpid creatures of my own fancy twitted me with imbecility, and not without fair occasion. (28)

Malgré sa véhémence, cette interpellation n'a rien de commun avec les agressions mentionnées précédemment. L'indignation des personnages apparait comme un juste rappel à l'ordre, et surtout comme un indispensable rappel à l'art. Cette mise en contact des créatures et de leur créateur crée chez ce dernier une prise de conscience salutaire, et l'immunise contre la mort artistique en réveillant sa vocation.

Les deux autres occurrences se trouvent à des moments décisifs et symétriques du roman - l'incipit (au troisième paragraphe) et la conclusion (à l'antépénultième paragraphe, c'est-à-dire le troisième paragraphe avant la fin). La métalepse du premier chapitre illustre la différence considérable qui sépare The Scarlet Letter de The Blithedale Romance: contrairement à Miles Coverdale, le narrateur affiche ici sa volonté de franchir les seuils narratifs. Dès le premier chapitre, il s'introduit dans le récit de façon spectaculaire.

This rose-bush [...] has been kept alive in history [...]. Finding it so directly on the threshold of our narrative, which is now about to issue forth from that inauspicious portal, we could hardly do otherwise than pluck one of its flowers, and present it to the reader. It may serve, let us hope, to symbolize some sweet moral blossom [...]. (37)

Sous les yeux du lecteur, le narrateur abandonne son statut extradiégétique pour s'introduire l'espace d'un instant dans la diégèse. Une fois ressorti de la diégèse, il s'immisce pendant une durée tout aussi infime dans la sphère du lecteur, auquel il offre la rose qu'il vient de cueillir. Malgré sa fugacité, cette double figure inaugurale fait partie intégrante de la signification globale du texte, et place le récit hawthornien sous le signe de la métalepse.

La dernière occurrence, plus discrète mais non moins importante, a lieu au chapitre XXIV :

In fine, the gossips of that day believed,-and Mr. Surveyor Pue, who made investigations a century later, believed,--and one of his recent successors in office, moreover, faithfully believes,-that Pearl was not only alive, but married, and happy, and mindful of her mother [...]. (165)

Le brouillage de la diégèse et de l'extradiégétique provient d'un nivellement narratif qui aligne des personnages situées dans l'illo tempore colonial (« the gossips of that day »), l'Inspecteur Pue (qui vécut au $18^{\text {ème }}$ siècle), et l'auteur des pages que nous lisons. 
Il relie ainsi trois époques historiques, le $17^{\text {ème }}$, le $18^{\text {ème }}$ et le $19^{\text {ème }}$ siècles. Mais surtout, il réunit deux niveaux ontologiques, l'histoire et la fiction (Pue d'une part, les personnages et le narrateur d'autre part), ainsi que deux niveaux narratifs distincts (la diégèse et l'instance énonciatrice). Il faut noter que, contrairement au chapitre initial qui mettait en relief le double franchissement métaleptique, cette quatrième métalepse se fait sous forme de glissement presque imperceptible. La porosité des frontières semble aller de soi, et s'accompagne d'une grande sérénité. Toute idée de transgression ou de violence disparaît.

Ces franchissements sont renforcés par la présence d'un autre élément. Même si les activités de Pearl ne sont pas de même nature, elles soulignent la stratégie métaleptique du roman. En effet, la fille de Hester est fréquemment associée au motif de la fenêtre, du miroir et du reflet dans la flaque d'eau :

[Pearl] escaped through the open window, and stood on the upper step, looking like a wild tropical bird, of rich plumage, ready to take flight into the upper air. (75)

[...]Pearl stood outside of the window [...]. (76)

[Pearl] had flirted fancifully with her own image in a pool of water, beckoning the phantom forth, and [...] seeking a passage into its sphere of impalpable earth and unattainable sky. Soon finding, however, that either she or the image was unreal, she turned elsewhere for better pastimes. (115)

La fenêtre par laquelle Pearl sort à plusieurs reprises a une valeur symbolique, et s'inscrit dans une tradition romantique, notamment picturale (on songe à Caspar David Friedrich, ou même à Hopper). Seuil entre l'intérieur et l'extérieur, la fenêtre représente souvent la pensée humaine, le désir du moi d'entrer en contact avec le monde extérieur. Son franchissement permet la communion intersubjective. Or la métalepse a une fonction analogue, bien qu'elle se situe sur un autre niveau. De plus, elle appartient à la même tradition esthétique, comme le rappelle sa première appellation, « ironie romantique ». Dans la mesure où les gestes de Pearl sont analogues à l'activité de l'écrivain, ils soulignent la nature métaleptique du récit, et font de Pearl la projection symbolique du narrateur hawthornien - contrairement à Dimmesdale, qui serait son repoussoir: incapable de respirer l'air frais, redoutant les fenêtres ouvertes, le jeune prédicateur est enfermé dans le cadre puritain ${ }^{10}$.

Puisque l'existence de ces "zones de contact» ne fait plus de doute, on peut maintenant chercher à interpréter leur signification. La métalepse est une trace parmi d'autres de l'hostilité du narrateur vis-à-vis de la société puritaine, exprimée par l'image récurrente du carcan de fer ${ }^{11}$. Il ne faut pas s'étonner si Hester est tentée de quitter sa chaumière délabrée et de repartir en Europe, ou si les protagonistes de The House of the Seven Gables décident, au chapitre XXI, de quitter « la sinistre maison aux sept pignons ». Ces bâtisses constituent des " édifices patriarcaux » (Tales and Sketches 1036), et symbolisent la loi tyrannique du père. Loi parfois synonyme de torture: l'arsenal répressif que constitue le pilori, machine de bois et de fer désignée par l'oxymore "ideal of ignominy " (The Scarlet Letter 42), suggère un sadisme latent. Les châtiments cruels sont également évoqués dans «Endicott and the Red Cross», et génèrent eux aussi tout un registre sadique (" encased ", " confined ", " a cleft stick on her tongue ", « ears cropped », « cheeks [...] branded », « nostrils slit and seared » Tales and Sketches 543). Pire, le carcan puritain cherche aussi à entraver l'esprit humain. L'herméneutique apocalyptique, qui ne tolère qu'une interprétation unique des signes, fige le sens - l'« empale ", pour reprendre l'image de la Préface de The House of the Seven Gables : 
The Author has considered it hardly worth his while, therefore, relentlessly to impale the story with its moral, as with an iron rod-or rather, as by sticking a pin through a butterfly-thus at once depriving it of life, and causing it to stiffen in an ungainly and unnatural attitude. (The House of the Seven Gables 2) récit, celle-ci permet au contraire la relation ${ }^{12}$, la porosité, l'échange. Elle est un des tropes fondamentaux de ce que Fleurdorge appelle le "contre-discours ironique » (Carlet 80). En subvertissant les frontières et les hiérarchies narratives, elle confère au texte une modernité, voire une postmodernité ${ }^{13}$ que d'autres figures ne sauraient lui conférer.

La critique de la loi puritaine s'explique par une aversion pour un pouvoir jugé abusif, et pour les discours contraignants qu'il génère. Ce pouvoir qui trace des frontières et impose des cadres (spatiaux, métaphysiques, discursifs) n'est pas sans rappeler le «pouvoir-savoir », défini par Michel Foucault. Si l'on essaie de recenser les « dispositifs disciplinaires » qui émaillent le récit hawthornien, de nombreux points de convergence avec les analyses de Foucault surgissent. Commençons par résumer brièvement certains concepts susceptibles d'éclairer The Scarlet Letter. Le pouvoir-savoir s'appuie, comme le montre Foucault, non pas sur l'art (ars), mais sur la «science » (scientia), qui est une "mise en discours» (Histoire de la sexualité 29). Au centre de ce discours de vérité se trouve l'aveu, un des «rituels majeurs dont on attend la production de la vérité » (Histoire 78), qui n'est jamais loin de la torture, son « noir jumeau » (Histoire 79). Ajoutons que Foucault évoque souvent le pouvoir en termes spatiaux, comme si celui-ci tendait à quadriller le domaine de l'humain, à imposer une "grille » inflexible (L'ordre $d u$ discours 11). « [II] introduit des discontinuités, il sépare ce qui est joint, il marque des frontières (Histoire 110). La description du "panoptisme", dans Surveiller et punir, est bien sûr un moment fondamental de cette démonstration. Le panoptique est un espace clos, centralisé, hiérarchisé, et «travers[é] de regard » (231). «Les murs empêchent tout contact » (234) entre les détenus; à l'extérieur, les citoyens forment non pas une foule, propice à des « échanges multiples ", mais une " collection d'individus séparés ", une " solitude séquestrée et regardée ». En somme, « la visibilité est un piège ».

Tous ces éléments se retrouvent à des degrés divers dans The Scarlet Letter. Sommée de passer à l'aveu, Hester subit un châtiment proche de la torture psychologique. Dans la foule assemblée, peu de véritables échanges : les matrones discutent, mais ne font que répéter la doxa puritaine, avec plus ou moins de cruauté. La seule femme qui, parmi elles, se montre compatissante, ne parvient pas à se faire entendre, et meurt à la fin du récit (156). Dans l'ensemble du roman, aucune véritable interaction entre les citoyens : ceux-ci semblent eux aussi isolés, séquestrés et regardés par le pouvoir. En ce sens, le châtiment d'Hester est ironique pour plusieurs raisons : il constitue une mise en scène théâtrale, ce qui est une première contradiction dans les termes dans une société censée condamner l'art dramatique. Mais l'ironie s'augmente du fait qu'il s'agit moins d'un «spectacle»-moment de "proximité sensuelle» où la société communie "comme un grand corps unique»-que d'une forme de "surveillance», qui est l'« inverse du spectacle ». Bref, le châtiment de Hester Prynne est «bien moins gre[c] que nous le croyons » (Surveiller et punir 252-3). Le villageois qui répond aux questions de Chillingworth (46) fait lui aussi entendre une doctrine puritaine parfaitement conforme, comme s'il redoutait d'être espionné. A l'instar du panoptique, la société puritaine semble induire « un état conscient et permanent de visibilité qui assure le fonctionnement automatique du pouvoir » (234) et empêche les contacts. 

pilori, lequel n'est pas sans rappeler la «tour centrale» des dispositifs panoptiques. Le Boston colonial ressemble ainsi aux "maisons de certitude " chères à Bentham (Surveiller 236), puisque tout est censé se ramener au discours de vérité. Les édiles puritains agencent l'espace en cercles concentriques : cercle du pouvoir, périmètre du village, périphérie où vit la pécheresse, forêt diabolique, océan-muraille qui sépare du Vieux Monde corrompu. Les colons cadrent, cadastrent et centralisent: à peine débarqués, il construisent une prison («prison-house » 36) et un cimetière («marked out the first burial-ground ", " the nucleus of all the congregated sepulchres in the old church-yard of King's Chapel » 36), et figent le langage à grands renforts de noms composés. Il n'est pas jusqu'à la lumière qui ne soit instrumentalisée: le soleil aveuglant de la place du marché où l'on traîne Hester Prynne symbolise le logos puritain, réputé infaillible. "A blessing on the righteous colony of the Massachusetts, where iniquity is dragged out into the sunshine!", s'exclame le bedeau (41). Pour Hester aussi, « la visibilité est un piège ».

En quoi la stratégie métaleptique déployée par Hawthorne propose-t-elle une alternative à ce discours de vérité ? Tout d'abord, le récit ne constitue pas une « mise en discours" s'appuyant sur la science, mais plutôt une mise en contact ayant pour médiation l'art. Il a pour figure tutélaire non pas le prophète, celui qui voit clairement - nul Daniel pour résoudre l'énigme («the Daniel who shall expound [this riddle] is yet a-wanting» 46) -, mais des figures artistiques (Pearl, Hester, Pue). L'ars, selon Foucault, s'appuie sur la pratique, l'expérience, et désigne un rapport au « savoir qui doit demeurer secret. [...] Le rapport au maître détenteur des secrets est donc fondamental ; seul celui-ci peut le transmettre sur le mode ésotérique et au terme d'une initiation où il guide [...] le cheminement du disciple ». La vérité livrée par cet art «n'est pas garantie par l'autorité hautaine du magistère [...] mais par le lien, l'appartenance essentielle dans le discours entre celui qui parle et ce dont il parle " (Histoire 77-84, nous soulignons). Certes, The Scarlet Letter se soucie de la vérité ( $« \mathrm{Be}$ true ! Be true ! Be true !»163), mais cette vérité est de nature empathique; c'est celle du cœur humain. Il s'agit moins de voir et de savoir (ce que cherchent à faire les puritains) que de percevoir et de ressentir de façon intuitive et ésotérique, grâce à la trans-mission. D'où l'importance des entrevues empathiques et ésotériques (diamétralement opposées aux entretiens, publics et privés, que les puritains et Chillingworth imposent à l'héroïne, et qui n'ont qu'un seul but, l'aveu) : $:^{14}$ entrevue du narrateur et de Pue, mise en contact du narrateur, de l'héroïne et du lecteur par la double métalepse initiale, et nouvelle mise en contact grâce au glissement métaleptique final. Le narrateur n'affirme plus sa supériorité afin de garantir son immunité, mais revendique l'hybridité des catégories narratives, et aspire à la contamination. La vulnérabilité fait partie intégrante de cette érotique de l'écriture et de la lecture. Ou, pour citer Dorrit Cohn: «Au moment où le narrateur se transforme en personnage fictif, il renonce à la position de supériorité et d'autorité sur son histoire ».

Le récit épouse donc une logique empathique, et non scientifique. Au lieu de nous renseigner sur Hester, au sens presque policier du terme, au lieu de mettre en place un «système d'enregistrement permanent» fondé sur un "travail ininterrompu d'écriture " (Surveiller 229-30), les métalepses établissent une relation trouble avec Hester. Trouble dans le sens où il n'y a plus de séparation absolue, mais où il n'y a pas davantage de transitivité totale ou de parfaite transparence. Le franchissement des 
barrières narratives joue par conséquent la même fonction que le voile hawthornien : il transforme les cadres étanches en sphères poreuses, et donne accès à un savoir secret, mais sans le profaner - la violation narrative n'est pas violation psychique. Cette fonction filtrante apparaît dès le début de "The Custom-House ", où le narrateur noue un pacte de lecture qui lui permet de s'adresser directement à son destinataire tout en voilant son moi le plus intime (7). Plus tard dans le récit, lors des retrouvailles des amants, la forêt fonctionne sur le même mode :

All these giant trees and boulders of granite seemed intent on making a mystery of the course of this small brook; fearing, perhaps, that, with its never-ending loquacity, it should whisper tales out of the heart of the old forest whence it flowed, or mirror its revelations on the smooth surface of a pool. (120, nous soulignons)

Si la forêt symbolise l'intimité secrète du moi, le ruisseau apparaît comme ce qui risque, par une verbalisation indiscrète, de la divulguer en cherchant à la mettre en discours et à la livrer aux regards. Telle un voile, la forêt donne accès au mystère du cœur humain, tout en sanctuarisant celui-ci. Dans un chapitre antérieur, les éléments naturels jouaient une fonction analogue. Les nuages flottant entre ciel et terre («the dense medium betwixt the sky and earth » [101]) forment non pas une limite infranchissable, mais une zone de contact, où il n'est pas interdit de voir un symbole réflexif de la perméabilité des niveaux narratifs. Enfin, la typographie est un dernier moyen de matérialiser la logique du récit. Citons à nouveau le passage suivant :

In fine, the gossips of that day believed,-and Mr. Surveyor Pue, who made investigations a century later, believed,-and one of his recent successors in office, moreover, faithfully believes,--that Pearl was not only alive, but married, and happy, and mindful of her mother [...]. (165)

Cet ultime glissement relie, par des tirets (et par la polysyndète), ceux qui connurent Hester, Jonathan Pue, et son "successeur ». En définitive, on voit que la métalepse hawthornienne évoque non pas l'image du clou, qui empale l'épistémologie et l'eschatologie, mais celle d'une chaîne. Contrairement à la «chaîne électrique » (101) que forment Dimmesdale, Pearl et Hester, qui est éphémère, précaire, et se situe dans la seule diégèse, celle-ci est une concaténation ${ }^{15}$ empathique et durable où se coalisent personnage, narrateur et narrataire. Cette châne autorise l'échange réciproque : si le narrateur s'introduit dans l'histoire, le personnage peut aussi sortir du cadre diégétique. Cette "double vectorisation» (Wagner 246) donne lieu à une véritable érotique, en donnant à eros tout son sens de manque et de quête ardente de réciprocité. En définissant la relation narrateur-lecteur comme une expérience fusionnelle susceptible de combler un sentiment d'incomplétude, le narrateur de "The CustomHouse $»^{16}$, prend des accents platoniciens dignes du Banquet. De ce fait, on peut affirmer, avec Agnès Derail-Imbert, que «[le] 'lettered intercourse' inaugural [...] occupe, dans l'économie narrative, la place du sexe illicite entre Hester et Dimmesdale [...]», même si, «[t]outefois, le rapport délictueux entre auteur et lecteur n'aura pas lieu » (Monfort [2005] 56). La possibilité d'une triangulation amoureuse entre narrateur et personnage, narrateur et lecteur, et, pourquoi pas, lecteur et personnage suggère le potentiel érotique de la métalepse. Elle accrédite également la thèse de Brian McHale, qui prend le problème dans le sens inverse, invoquant l'inhérence de la métalepse au thème amoureux :

Love [...] makes fiction go round [and l]ove as a principle of fiction is, at least in two senses, metaleptic. If authors love their characters, and if texts seduce their readers, then those relations involve the violation of ontological boundaries. [...] 
These metaleptic relations are permanent features of modern western literature [...]. (Malina 5-6) personnages et celle du narrateur ne sont que le reflet l'une de l'autre. Si la métalepse fait du narrateur le partenaire d'Hester dans cette chaîne érotique, elle constitue une duplication de l'adultère. L'art (métaleptique) et l'adultère sont les deux transgressions majeures auxquelles renverrait la lettre écarlate.

\section{Conclusion} types d'expériences fondamentales: le sacré et l'énigme. Une telle affirmation peut sembler doublement paradoxale : comment la métalepse peut-elle dans le même temps renvoyer au sacré si, par ailleurs, elle subvertit la piété puritaine? De plus, si la elle franchit une "frontière mouvante mais sacrée », comme l'écrit Genette, n'est-elle pas ipso facto sacrilège? Il faut d'abord préciser ce que l'on entend par sacré. La vision puritaine sépare de façon binaire, dissocie le Bien et le Mal, le divin et le maudit. De même que les concepts "puritain" et "pur", impliquent la démarcation d'avec l'impur, le sacré présuppose souvent la séparation. Giorgio Agamben résume ainsi cette première conception du religieux :

On peut définir la religion comme ce qui soustrait les choses, les personnes à l'usage commun pour les transférer dans une sphère séparée. Non seulement il n'est pas de religion sans séparation, mais toute séparation contient ou conserve par-devers soi un noyau authentiquement religieux. Le dispositif qui met en œuvre et qui règle la séparation est le sacrifice : il marque [...] le passage de la sphère du profane au sacré [...]. La césure qui sépare les deux sphères est essentielle. (Profanations 96)

Dans The Scarlet Letter, cette logique de la séparation aboutit d'un côté à sanctifier Winthrop, et de l'autre à faire de la femme adultère l'incarnation du péché («the reality of sin » 55). Impure, Hester est mise à l'écart et fait peur (« a strange, contagious fear » 57).

Or on a vu que la métalepse permettait de rompre avec une telle conception. Elle instaure un régime généralisé d'indifférenciation fondé sur la réversibilité du diégétique et de l'extradiégétique. Si la métalepse est la figure de l'indifférenciation ontologique, elle peut rejoindre la notion de sacré, à condition que l'on revienne à ce qui constitue, d'après Giorgio Agamben, la définition primitive du terme. Le sacré désigne à l'origine non pas ce qui sépare, mais justement l'ambigu et l'indifférencié. Agamben met bien en évidence cette absence de démarcation primordiale entre sainteté et impureté, horreur et vénération ${ }^{17}$, Rudolf Otto notait déjà que le sentiment numineux est ambigu, puisqu'il va "de la quiétude à l'extase, en passant par le saisissement et l'horreur » (Le sacré 36). La crainte qu'inspire Hester est donc tout aussi sacrée que l'admiration vouée à Winthrop. Par ailleurs, la métalepse explique également l'importance de l'énigme, thème récurrent dans le roman ${ }^{18}$, car l'énigme est la «conjonction des opposés» (Homo sacer 40). Si le sacré est une mise en contact psychologique et eschatologique où se mêlent peur et vénération, ainsi que bien et mal, si l'énigme est une mise en contact épistémologique d'un sens et d'un sens opposé, la spécificité de l'écriture hawthornienne ne doit plus surprendre. The Scarlet Letter est un récit, troublant et non violent, qui efface les démarcations de toutes sortes. C'est le récit de l'indétermination épistémologique, eschatologique et ontologique.

Loin d'être sacrilège, le trope joue une fonction religieuse, dans la mesure où il relie les instances narratives, et salvatrice, car il brise la solitude de Hester et du narrateur. On 
objectera peut-être que Hester, dont la dépouille est finalement enterrée dans le cimetière puritain ("that burial-ground beside which King's Chapel has since been built » 166), rentre finalement dans le cadre de la loi en retrouvant Boston et la lettre écarlate - mais c'est sans compter avec l'épitaphe: «On a field, sable, the letter A, gules». Dans cette inscription, la lettre se détache sur une toile de fond ouverte. Ni empalée, ni assujettie, elle est simplement en contact, ayant toute latitude pour se déplacer ou s'absenter. Le récit métaleptique a transformé le cadre en champ ouvert.

AGAMBEN, Giorgio, Homo sacer. Le pouvoir souverain et la vie nue. [1995]. Trad. Marilène Raiola (Paris : Éditions du Seuil, 1997).

---, Profanations. [2005]. Trad. Martin Rueff (Paris : Éditions Payot et Rivages, 2006).

AMFREVILLE, Marc et Antoine CAZÉ, dir., The Scarlet Letter. Nathaniel Hawthorne (Paris : Ellipses, 2005).

BAYM, Nina, The Scarlet Letter: A Reading (New York : Twayne Publishers, 1986).

--- , « The Major Phase I : 1850 ». Nathaniel Hawthorne, The Scarlet Letter, Rita K. Gollin, ed. (Boston : Houghton Mifflin Company, 2002), 332-349. BEECHER STOWE, Harriet, Three Novels (New York : The Library of America, 1982). BORGES, Jorge Luis, Enquêtes. [1957]. Trad. Paul et Sylvia Bénichou (Paris : Gallimard, coll. Folio/essais, 1986).

BRODHEAD, Richard H., The School of Hawthorne (New York: Oxford University Press, 1989).

51 CARLET, Yves, textes réunis par, Profils américains « Nathaniel Hawthorne. Les contes » 1 (1991).

COHN, Dorrit, "Métalepse et mise en abyme ». http://www.vox-poetica.org/t/ metalepse.htm: Vox Poetica, 2003.

FOUCAULT, Michel, L'ordre du discours. Leçon inaugurale au Collège de France prononcée le 2 décembre 1970. [1971] (Paris : Gallimard, 2004).

54 ---, Surveiller et punir. Naissance de la prison. [1975] (Paris : Gallimard, coll. « Tel », 2004).

55 ---, Histoire de la sexualité. 1 La volonté de savoir. [1976] (Paris : Gallimard, coll. «Tel», 2005).

GENETTE, Gérard, Figures III (Paris : Éditions du Seuil, 1972).

57 ---, Nouveau discours du récit (Paris : Éditions du Seuil, coll. « Poétique », 1983).

58 ---, Gérard, Métalepse. De la figure à la fiction (Paris : Éditions du Seuil, 2004).

59 HAWTHORNE, Nathaniel, The Complete Novels and Selected Tales, Norman Holmes Pearson, ed. (New York : The Modern Library, 1937).

$60---$, The House of the Seven Gables. [1851] (New York: W. W. Norton \& Company, 1967).

61 ---, Tales and Sketches (New York : The Library of America, 1996).

62 ---, The Scarlet Letter, Rita K. Gollin, ed. (Boston : Houghton Mifflin Company, 2002).

63 ---, The Scarlet Letter and Other Writings (New York: W.W. Norton \& Company, 2005).

64 JAWORSKI, Philippe, textes réunis par, Hawthorne et la pensée du roman. Dix études sur La Lettre écarlate (Paris : Michel Houdiard Éditeur, 2006).

65 KAUL, A. N., ed., Hawthorne: A Collection of Critical Essays (Englewood Cliffs : PrenticeHall, 1966). 
MALINA, Debra, Breaking the Frame: Metalepsis and The Construction of the Subject (Columbus : Ohio, 2002).

MILLINGTON, Richard H., ed., The Cambridge Companion to Nathaniel Hawthorne (Cambridge : Cambridge University Press, 2004).

MONFORT, Bruno, Nathaniel Hawthorne, Contes et Nouvelles. Le territoire du presque (Paris : Ellipses, 2000).

---, dir., Nathaniel Hawthorne. The Scarlet Letter (Paris: Editions du Temps, 2005).

MOORE, Thomas R., «A Thick and Darksome Veil: The Rhetoric of Hawthorne's Sketches », Nineteenth-Century Literature 48 : 3 (décembre 1993), 310-325.

NORMAND, Jean, Nathaniel Hawthorne. Esquisse d'une Analyse de la Création Artistique (Paris : Presses Universitaires de France, 1964).

OTTO, Rudolf, Le sacré. L'élément non rationnel dans l'idée du divin et sa relation avec le rationnel. [1949]. Trad. André Jundt (Paris: Editions Payot et Rivages, coll. «Petite bibliothèque Payot », 2001).

SIMONSON, Patricia, «L'ambivalence de la prise de parole dans l'écriture de Nathaniel Hawthorne: le dilemme de Jonas ». Thèse de Doctorat, dir. Hubert Theyssandier, Université Paris III-Sorbonne Nouvelle, 1998.

THOMPSON, G. R., " Literary Politics and the 'Legitimate Sphere' : Poe, Hawthorne, and the ‘Tale Proper' ", Nineteenth-Century Literature $49: 2$ (septembre 1994), 167-195.

WAGNER, Frank, "Glissements et déphasages. Note sur la métalepse narrative ", Poétique 130 (avril 2002).

WAGSTAFF, Sheena, ed., Edward Hopper (Londres : Tate Publishing, 2004).

\section{NOTES}

1. Simonson 23, Monfort (2000), Baym (Gollin 346), Roudeau (Monfort [2005] 28-29), Derail-Imbert (Montfort [2005] 46), Jaworski (Carlet 115), Amfreville et Cazé 5.

2. À notre connaissance, la métalepse n'est mentionnée que par trois critiques, Claude Fleurdorge (Carlet 83), Hélène Guillaume (Amfreville et Cazé 55), et Antoine Cazé (Jaworski 16).

3. « [T] he artistic exploitation of metalepsis has run rampant in the postmodern era of the collapse of master narratives, the dismantling of the category of the real, and the deconstruction of binary and hierarchical systems of understanding. " (Malina 1-2) « Even at its mildest, metalepsis disrupts the boundary of a fictional narrative-the one between inside and outside, between story and world. When a text repeatedly indulges in such subversion, the result is inevitably jarring, and its effects run the gamut between from startling diversion through destabilization and disorientation to outright violation. If I emphasize a violent streak underlying this persistent breaching of constitutive boundaries, it is because I detect, even in the a certain game or joke, a certain aggression toward the subject, whether internal or external to the text. [...] [Many authors indulge] in metalepses designed to undermine the reader's security 
in their exclusively extratextual existence and their certainty about the separation between life and literature. » (Malina 3-7)

4. « Let us enter the dwelling. [...] Just at present, however, Aunt Chloe is looking into the bake-pan ; in which congenial operation we shall leave her till we finish our picture of the cottage. » (Three Novels 32-33)

5. «Ah, I perceive what you are about! You are turning this whole affair into a ballad. Pray let me hear as many stanzas as you happen to have ready. [...] It is a genuine tragedy, is it not? [...]. But, Mr. Coverdale, by all means write this ballad, and put your soul's ache into it, and turn your sympathy to good account, as other poets do, and as poets must, unless they choose to give us glittering icicles instead of lines of fire. »

6. « Yet I felt a hesitation about plunging into this muddy tide of human activity and pastime. It suited me better, for the present, to linger on the brink, or hover in the air above it. [...] My book was of the dullest, yet had a sort of sluggish flow, like that of a stream in which your boat is as often aground as afloat. Had there been a more impetuous rush, a more absorbing passion of the narrative, I would the sooner have struggled out of its uneasy current [...]. » (526)

7. Dans The Scarlet Letter, le manuscrit de Jonathan Pue connaît le même sort : il fait partie du récit de la découverte de la lettre écarlate (qui constitue la diégèse de «The Custom-House »), puis passe dans la sphère narrative : " The original papers, together with the letter itself,-a most curious relic,-are still in my possession, and shall be freely exhibited to whomever, induced by the great interest of the narrative, may desire a sight of them » (27, nos italiques). Cette métalepse va plus loin que celle de The Blithedale Romance, puisque le manuscrit franchit virtuellement un autre seuil narratif, en passant dans la sphère du lecteur. Ce geste annonce celui du narrateur qui, au chapitre I, offre au lecteur extradiégétique une rose cueillie dans la diégèse.

8. « '[...] hold your tongue [...]' » (576), « indignation. » (578)

9. Il remarque lui-même : « That cold tendency, between instinct and intellect, which made me pry with a speculative interest into people's passions and impulses, appeared to have gone far towards unhumanizing my heart. [...] To escape the irksomeness of these meditations, I resumed my post at the window. » (530)

10. «It was as if a window were thrown open, admitting a freer atmosphere into the close and stifled study, where his life was wasting itself away [...]. But the air was too fresh and chill to be long breathed with comfort. So the minister, and the physician with him, withdrew again within the limits of what their church defined as orthodox » (83). « As a priest, the framework of his order inevitably hemmed him in » (128-9).

11. Dans The Scarlet Letter, on trouve les expressions suivantes : « iron framework » (83, 106) ; « iron arm » (55); « iron links » (56), « iron men » (127). La même image revient dans « Main-street».

12. Le champ lexical de la mise en relation est récurrent : « connected » (22), « disconnect » (35), « connected » (56), « to connect » (61); intercourse (64); « connecting link» (101); « a meeting-point of sympathy » (116); « intimately connected in their former life » (122); « the tie that united them » (132); « no real bond » (152); « connected with this spot » (155).

13. «In the very traditional, yet characteristically romantic, fusion of the dramatized and presentational narrative with the discursive voice and personality of an author or author-figure, Hawthorne's nineteenth-century narratives bear interesting resemblances to twentieth-century 'postmodernist' fiction, in particular to that of John 
Barth in such 'story' collections as Lost in the Funhouse (1968) and Chimera (1972) ». (Thompson 193)

14. La résistance du texte à la logique de l'aveu apparaît aussi dans le « maniement ironique de l'aveu » dont parle Agnès Derail-Imbert (Montfort [2005] 41).

15. Hawthorne est donc, à plus d'un titre, le « concaténateur » dont parle Richard Brodhead: « Paul Elmer More's Sherburne essays on Hawthorne, the finest Hawthorne criticism of the turn of the century, treat him [...] as a missing link, establishing the continuity between such ancestors as Cotton Mather and such writers of the present as Mary Wilkins Freeman. Within such studies Hawthorne functions as the concatenator, the author who pulls disparate writing into a coherent and continuous line " (Brodhead 9). Son œuvre constitue le chaînon manquant entre plusieurs tendances littéraires, comme le montre Brodhead, mais cherche aussi à rapprocher les niveaux narratifs au sein du texte, comme le montre la tendance métaleptique. Sur le plan intertextuel de l'histoire littéraire, et sur le plan intratextuel des stratégies narratives, elle est placée sous le signe de la contiguïté.

16. «Some authors, indeed, do far more than this, and indulge themselves in such confidential depths of revelation as could fittingly be addressed, only and exclusively, to the one heart and mind, of perfect sympathy; as if the printed book, thrown at large on the wide world, were certain to find out the divided segment of the writer's own nature, and complete his circle of existence by bringing him into communion with it " (7).

17. « [U]n signifiant flottant passe [d'un pôle] à l'autre sans cesser de se rapporter au même objet » (Profanations 103). " 'A côté de certains tabous, écrit Robertson Smith [...], qui correspondent exactement à des règles de sainteté [...], on rencontre une autre espèce de tabou qui, dans la culture sémitique, trouve un parallèle dans les règles d'impureté. [...] Dans nombre de sociétés primitives il n'existe aucune ligne de démarcation entre ces deux tabous, et même chez certains peuples évolués la notion de sainteté et celle d'impureté sont souvent contiguës'. [L]e concept de tabou exprime l'indifférenciation originaire entre le sacré et l'impur, [...] ce mélange de vénération et d'horreur que Wundt [...] définit comme 'horreur sacrée'. C'est seulement dans une phase ultérieure [de la pensée humaine, selon Wundt], que cette ambivalence originaire fit place à l'antithèse du sacré et de l'impur. [...] 'Le pur et l'impur [selon Emile Durkheim] ne sont pas deux genres séparés, mais les deux variétés d'un même genre qui comprend toutes les choses sacrées. Il y a deux sortes de sacré, l'un faste, l'autre néfaste [...] un même objet peut passer de l'un à l'autre sans changer de nature. C'est dans la possibilité de ces transmutations que consiste l'ambiguïté du sacré' » (Homo sacer 86-8).

18. Voir « riddle » $(26,46)$, « enigma » (117).

\section{AUTEUR}

\section{GUILLAUME TANGUY}

Université Paul Valéry-Montpellier III 